\title{
Perioperative Pain Correlates and Prolonged Postoperative Pain Predictors: Demographic and Psychometric Questionnaires
}

\author{
Campbell MacLachlan · Edward A. Shipton · J. Elisabeth Wells
}

To view enhanced content go to www.paintherapy-open.com

Received: April 1, 2015 / Published online: June 3, 2015

(C) The Author(s) 2015. This article is published with open access at Springerlink.com

\begin{abstract}
Introduction: Perioperatively, patients are near-guaranteed to experience acute pain by virtue of the surgical tissue insult. The transition of acute pain to pathological chronic pain is a complex and poorly understood process. To study this, the prevalence of pain was examined preoperatively, and at 6 weeks and 3 months postoperatively.
\end{abstract}

Methods: Fifty-four patients undergoing moderate-major gynaecological surgery at Christchurch Women's Hospital (Christchurch, New Zealand) were recruited over a period of 11 weeks. Follow-up by telephone was conducted at 6 weeks and 3 months following

Electronic supplementary material The online version of this article (doi:10.1007/s40122-015-0037-7) contains supplementary material, which is available to authorized users.

C. MacLachlan · E. A. Shipton ( $\square)$

Department of Anaesthesia, University of Otago, Christchurch, New Zealand

e-mail: shiptonea@xtra.co.nz

\section{J. E. Wells}

Biostatistics and Computational Biology,

Department of Population Health, University of

Otago, Christchurch, New Zealand surgery. Demographic information including age, gender, ethnicity, work, and education status were collected, as well as aspects of medical history. Participants were subjected to psychometric questionnaires at each time-point. Results: Of the participants, $15.7 \%$ experienced significant pain at 6 weeks postoperatively; $8.2 \%$ of participants experienced significant pain at 3 months postoperatively. The psychometric questionnaires used found differences between those experiencing pain and those not experiencing pain at given observation points. Only the Brief Illness Perception Questionnaire (BIPQ) appeared predictive of developing prolonged postoperative pain. The mean difference (7.4 on a 0-50) scale should assist in clinical decision-making regarding analgesia.

Conclusion: Only the BIPQ was predictive of developing prolonged postoperative pain. While none of the demographic factors observed significantly predicted the development of 'prolonged pain', the not significant data followed expected trends. Several relationships were detected in this study that should further efforts in developing preoperative predictors to promote the 
secondary prevention of postoperative pain states.

Keywords: Pain; Postoperative; Prolonged; Demographics; Psychological factors

\section{INTRODUCTION}

Postoperative pain is mainly the result of tissue damage that causes the release of inflammatory chemicals, further sensitizing surrounding nociceptors [1]. Chronic pain is loosely defined as pain 'persisting beyond the accepted period of healing' [2]. However, in practice it is most often arbitrarily labeled as pain lasting longer than three or 6 months [3, 4]. The transition of acute pain to pathological chronic pain is a complex and poorly understood process.

Chronic post-surgical pain (CPSP) can be expected to occur in 5-60\% of surgeries [5-7]. It results in individual suffering $[8,9]$ and has an economic impact as well $[7,10]$. Chronic pain as a whole affects $16.9 \%$ of New Zealanders [11], and $20 \%$ and $17 \%$ of Australian females and males, respectively [12]. The 1-year prevalence of adult CPSP typically ranges between 1.5\% and $10 \%$ [13]. Certainly in the United Kingdom, surgery is the second most common reason given for having developed chronic neuropathic pain [14]. One to three percent of patients with CPSP will suffer from severe pain and pain-related interference with daily activities [15].

'Subacute' pain is a concept poorly defined in the literature, being loosely seen as term used to describe the stage between 'acute' pain and 'chronic' pain such as acute persistent pain [5]. For the purposes of this study, 'subacute' pain has been arbitrarily defined as 'pain at 6 weeks following surgery', as a halfway mark between 'acute' and 'chronic' pain (3 months). Little work appears to have been published on the pathophysiological changes at this stage.

Psychological factors such as depression and anxiety [16, 17] as well as demographic information such as age [18] and gender [19] are known to correlate with perioperative pain, and the development of CPSP following surgery.

It is hypothesized that one or more of the proposed factors measured perioperatively would predict continued pain at 6 weeks and 3 months after gynecological surgery. The objectives of the study were to determine the prevalence of acute persistent pain at 6 weeks following surgery; to determine the prevalence of chronic pain at 3 months following surgery; and to determine the extent to which (if at all) the perioperative factors measured predict continued pain at 6 weeks and 3 months.

\section{METHODS}

\section{Methods and Participant Flow}

This prospective cohort study was undertaken using a group of patients $(n=54)$ undergoing moderate to major gynecological surgery at Christchurch Women's Hospital, Christchurch, New Zealand. Surgical groups included: excision of endometriosis; hysterectomy; hysterectomy and salpingectomy and/or oophorectomy; salpingectomy and/or oophorectomy; pelvic floor/vaginal wall repair; and other surgeries. The study aimed to estimate the prevalence of pain at 6 weeks and 3 months postoperatively, and to determine the predictive value, if any, of the secondary measures taken. This paper focusses on the psychometric questionnaires 
Table 1 Psychometric questionnaires used at each follow-up point

\begin{tabular}{llll}
\hline Psychometric questionnaires used & Baseline (preadmission) & $\mathbf{6}$ weeks postoperatively & $\mathbf{3}$ months postoperatively \\
\hline Depression anxiety stress scale-21 & All patients & All patients & All patients \\
Brief illness perception questionnaire & All patients & None & None \\
Pain treatment satisfaction scale & None & All patients & None \\
Short-form McGill pain questionnaire & Patients in pain & Patients in pain & Patients in pain \\
\hline
\end{tabular}

administered, as well as the medical history and demographic information collected.

Inclusion criteria were as follows: those 16 years of age or older; those undergoing gynecological surgery requiring at least an overnight stay at Christchurch Women's Hospital, or Southern Cross Hospital in Christchurch; and those granting informed consent. Potential participants were excluded if they were: under 16 years of age; cognitively impaired (failing a person, place, and time orientation test); deemed not to be proficient in English to answer the questionnaires by phone; or did not grant informed consent. Patients undergoing oncological-gynecological surgery were not formally excluded. However, at the request of the preadmission clinic nursing staff none of these were approached by the investigators. Participants were grouped by surgical groups according to their primary indication for surgery or their underlying pathology.

Ethical approval was obtained from the University of Otago Human Ethics Committee (Health), and from the University of Otago, Christchurch Māori Research Advisor. Patients were approached at the Christchurch Women's Hospital preadmission clinic, where the study was explained to them, and written informed consent obtained if interest shown in participating in the study. Following this, psychometric questionnaires were completed, and the demographic information collected.
Whether or not a participant was experiencing subjectively impairing or distressing pain was noted. Many of the 'in pain' group could have been undergoing surgery to relieve longstanding pain. This was defined as either a 'usual pain' on the Visual Analogue Scale (VAS) of 3 or greater, or 'highest pain' on the VAS of 5 or greater, or if the participant verbally described significant distress or functional impairment. This assessment was carried out no more than $72 \mathrm{~h}$ preoperatively. Telephone follow-up was conducted at 6 weeks and 3 months postoperatively, where participants were again questioned about their pain status, and completed psychometric questionnaires. The questionnaires used at each time-point, and for which groups are shown in Table 1.

\section{Demographic, Clinical History, and Psychometric Questionnaires}

The demographic, clinical history, and psychometric questionnaires were administered together at the baseline assessment in Christchurch Women's Hospital Preadmission Clinic, under the supervision of one of the investigators trained to administer them.

The questionnaires chosen were selected because of their proven relevance to pain. All had been previously validated and proven to be robust, enabling a wide range of psychological factors to be analyzed. The short forms of many questionnaires were chosen for the sake of 
practicality. Lengthy telephone calls would place a time burden on our participants. As such, the short-forms of questionnaires were chosen wherever possible and practical. Each questionnaire is briefly described in Appendix I in the electronic supplementary material.

\section{ANALYSIS}

Data were entered into Microsoft Excel (Microsoft Corporation, Ann Arbor, MI, USA) spreadsheets, then into OpenEpi (Dean A.G. et al. Open Source Epidemiologic Statistics for Public Health) for quantitative analysis. The 95\% confidence intervals (95\% CI) were calculated using the Score (Wilson) method. $T$ tests (dependent and independent), and Chi square tests were performed as required.

\section{RESULTS}

Fifty-four participants were recruited, and underwent the baseline assessment. The nature of the clinical environment made it impossible to know for certain how many potential participants declined to participate or were not properly approached. At the 6-week follow-up point, three participants were considered lost to follow-up after they were unable to be contacted despite multiple attempts. All three of these participants had been 'in pain' at the baseline assessment. A further two participants were lost to follow-up at the 3-month follow-up point, again being unreachable by telephone. Neither of these participants had been 'in pain' at 6 weeks, although one was 'in pain' at baseline. Overall, four of the five participants lost to the study had been 'in pain' at baseline. The percentage in pain at baseline did not differ significantly between those who dropped out and those who did not $(P=0.187)$.

Fifty-one participants remained for the 6-week analysis, and 49 for the 3-month analysis. Participant flow is shown diagrammatically in Fig. 1.

\section{Prevalence of Pain at Each Follow-up Point}

At baseline, 28 (51.9\%) of the cohort $(n=54)$ were suffering from clinically significant and constant pain. At 6 weeks postoperatively, 8 $(15.7 \%)$ of the 51 remaining participants, and at 3 months postoperatively $4(8.2 \%)$ of the 49 remaining participants were suffering from clinically significant and constant pain. For the sake of analysis due to the small total sample size $(n=54)$, those 'in pain' at 6 weeks postoperatively were combined with those 'in pain' at 3 months postoperatively to give a single 'prolonged pain' group $(n=11)$. Seven

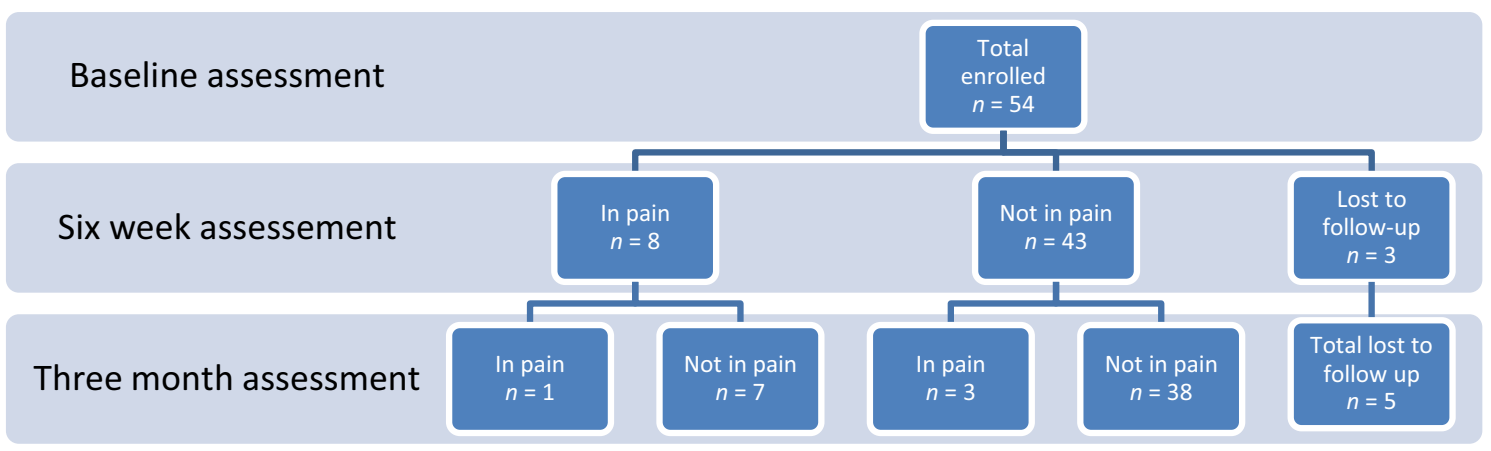

Fig. 1 Participant flow. All three participants lost to follow-up at 6 weeks had been 'in pain' at baseline. Both participants lost to follow-up at 3 months had been pain-free at 6 weeks although one had been in pain at baseline 
(63.6\%) of the 11 participants that developed 'prolonged pain' had suffered pain at baseline, compared to 21 (48.9\%) of the remaining 43 participants not 'in pain' at baseline. As this difference was not significant $(P=0.379)$, many of the analyses did not require control of the baseline pain.

\section{Psychometric Questionnaires}

Table 2 shows the baseline Depression Anxiety Stress Scale-21 (DASS-21) and Brief Illness Perception Questionnaire (BIPQ) scores for the following: those not 'in pain' at baseline; those 'in pain' at baseline; those who would not go on to develop 'prolonged pain'; and those who would go on to develop 'prolonged pain'. The published norms are given for comparison. These seem to have been gathered from participants in different circumstances, and in overseas locations that may have created subtle cultural differences.

\section{DASS-21}

At baseline those 'in pain' scored significantly higher on all three DASS-21 sections and overall as well. The cohort at 3 months scored lower than it had at 6 weeks (mean difference 4.7) and at baseline (mean difference 7.9). This reflected a true shift in the proportion of the cohort that was 'in pain', and the resulting effect on affective scores. The 'not in pain' group at 3 months scored lower on every sub-scale than either of the previous 'not in pain' groups. This would indicate the change in the proportion of the cohort 'in pain' was not the sole factor underlying improved affective scores.

Baseline DASS-21 scores were analyzed to see if they had significantly differed between those who would go on to develop 'prolonged pain' and those who would not. No significant differences were found despite the 'prolonged pain' group scoring higher on all three subscales (and overall) at baseline (depression $P=0.344$, anxiety $P=0.121$, stress $P=0.617$, total DASS-21 score $P=0.263$ ).

\section{BIPQ}

The mean BIPQ score in the group 'in pain' at baseline (41.1) was significantly higher $(P=0.003)$ than the group 'not in pain' at baseline (33.4). This indicated a more threatening view of illness in those 'in pain', in keeping with their higher anxiety and stress scores. When the BIPQ scores from the baseline assessment were compared between the 'prolonged pain' group and the rest of the cohort, BIPQ scores were significantly higher in the 'prolonged pain' group (mean difference 7.4, $P=0.049$ ).

No published normal values for the Causal Categories section of the BIPQ could be found from groups that were comparable to this cohort. To perform formal statistical analyses on this section was difficult, as many participants gave two or fewer responses, and their responses had to be arranged into somewhat arbitrary groups. Twelve (19.7\%) participants believed their gender or genetics caused their illness; 12 (19.7\%) answered, but stated they had 'no idea' of the cause of their illness; 10 (16.4\%) blamed their lifestyle or other self-controlled factors; for their illness; 9 (14.8\%) blamed childbirth for their illness; 9 (14.8\%) blamed other assorted biological factors for their illness; 6 (9.8\%) believed their age had a role to play in their illness; and 3 (4.9\%) believed their illness was caused by chance or by poor luck. Ten (18.5\%) of the 54 participants left this section of the BIPQ unanswered. Those 


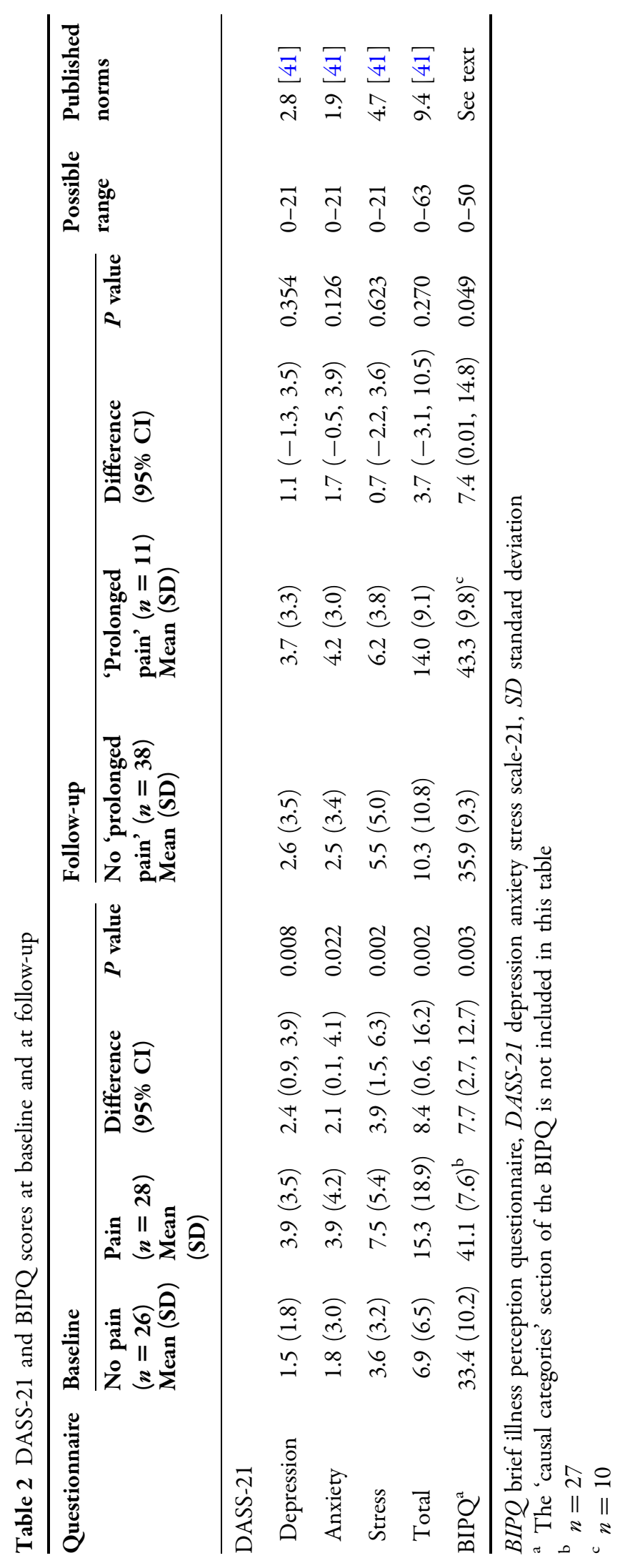


lost to follow-up were not excluded from analyses unrelated to pain outcomes.

\section{Pain Treatment Satisfaction Scale}

The pain treatment satisfaction scale (PTSS) was administered at the 6-week postoperative follow-up point. The mean score, out of 50, for those not 'in pain' at 6 weeks $(n=43)$ was 44.9 (SD 5.7). For those 'in pain' at 6 weeks postoperatively $(n=8)$, the mean score was 42.3 (SD 6.8). This difference was not significant $(P=0.336)$. When the 'prolonged pain' group $(n=11)$ was compared to the rest of the 6-week cohort $(n=40)$, the result remained non-significant $[43.7$ (SD 6.4) vs. 44.6 (SD 5.9), $P=0.681]$. Both groups answered in a similar manner to the 'as expected' question $(P=0.610)$ and to the 'would recommend' question $(P=0.289)$. A total of three participants stated that their treatment was not what they expected. Five indicated that they would not recommend the treatment.

\section{Short-Form McGill Pain Questionnaire}

Of the eight participants who were identified as experiencing clinically significant pain at 6 weeks following their surgery, six participants had been classified as 'in pain' at the baseline assessment. The Short-Form McGill Pain Questionnaire (SF-McGill) scores collected at 6 weeks were, overall, higher than those collected at baseline. The mean difference in the sensory scale was 7.8 , in the affective scale 0.7 , and for the overall score, 8 . Their SF-McGill scores on the sensory scale, but not the affective scale, were significantly higher $(P<0.001$ and $P=0.41$, respectively) to their baseline scores. This suggests that the pain experienced by these participants was unlikely to have simply been a symptom of their underlying condition.

\section{Demographic Information and Clinical History: Baseline Distribution and Predictive Value}

All 54 participants self-identified as female, nullifying any analysis relating to gender. Those 'in pain' at baseline are hereafter referred to as 'IP', and those not 'in pain' at baseline are hereafter referred to as 'NP'.

\section{Ethnicity}

Both groups had similar distribution of ethnicities, with the predominant selfidentified ethnicity being New Zealand European (81.5\%). As the 2013 New Zealand Census allowed multiple ethnicities [20], the two participants who selected two ethnicities had both recorded. The sample looked representative of the Canterbury region. The 2013 New Zealand Census reported New Zealand Māori as comprising $8.1 \%$ of the Canterbury region, New Zealand European as comprising $86.9 \%$ of the Canterbury region, and other ethnicities as comprising $14.1 \%$ the Canterbury region. Of the 'prolonged pain' group $(n=11), 9(81.8 \%)$ self-identified as New Zealand European, and 2 (18.2\%) selfidentified as other ethnicities. None selfidentified as New Zealand Māori.

Age

The youngest participant in the study was 20 years old, and the oldest was 73 years old. The mean age of those 'in pain' at baseline (36.3, SD 10.7) was 12.1 years lower than those not 'in pain' (48.4, SD 12.0; $P=0.001$ ). Age was not predictive of 'prolonged pain', as the 'prolonged pain' group's mean age (43.8, SD 11.5) was not substantially or significantly different from the rest of the cohort (41.7, SD $13.5 ; P=0.610)$. 


\section{Relevant History}

A history of chronic pain, defined as 'pain lasting longer than 3 months', was reported by 27 (50\%) of the participants at baseline. Those 'in pain' at baseline were significantly more likely to self-report a history of chronic pain $(P=0.007)$. This is not surprising, as many of the IP group could have been undergoing surgery to relieve longstanding pain. Those in the 'prolonged pain' group were more likely to have a self-reported history of chronic pain at baseline, but this was not significant $(P=0.313)$. The prevalence of chronic pain in the underlying New Zealand population is $16.9 \%$ [11].

A history of mental illness seems likely to be under-reported on a simple self-reporting questionnaire, due to embarrassment or inaccurate recall. Thirteen participants $(24.1 \%)$ reported a prior or current mental illness; this was higher in the IP group than in the NP group. This was not significant $(P=0.15)$. Both groups individually (and in total) were lower than the lifetime prevalence for 'any disorder' (39.5\%) found in Te Rau Hinengaro, the New Zealand Mental Health Survey [21]. This survey specifically enquired about symptoms of many disorders and was more sensitive to a history of mental illness than the question used in this study. Similarly, those in 'prolonged pain' were not significantly more likely to have reported a history of mental illness than the rest of the cohort $(P=0.779)$.

With the category of 'surgery' being broad, it has been difficult to find lifetime risk reports in the literature, particularly for New Zealand. One study from the United States reports a risk of intra-abdominal surgery by the age of 60 to be $43.8 \%$ [22], while two other studies report female lifetime risks of prolapse and urinary stress incontinence surgery to be 19\% [23] and $20 \%$ [24], respectively. With a mean age of only
41.5 years, the women in this study were not representative of a 'lifetime' risk. With 37 participants (68.5\%) having surgical histories, previous surgery was associated with risk of further surgery. Participants in the IP group were not significantly more likely than those in the NP group to have previously undergone surgery $(60.7 \%$ vs. $76.9 \%, P=0.20)$. In addition, the 'prolonged pain' group was not significantly more likely to report previous surgery than the rest of the cohort $(81.8 \%$ vs. $61.5 \%, P=0.289)$.

\section{Smoking, Alcohol Consumption, and Recreational Drug Use}

Those developing 'prolonged pain' were more likely to have smoked than those who did not, but this trend was not significant $(54.5 \%$ vs. $35.6 \%, P=0.298)$. For some participants recall bias may have affected reporting of the duration of smoking history. It is not known how this affected the overall results. Of the total participants, $40.8 \%$ of the total participants identified themselves as current (20.4\%) or former (20.4\%) smokers. The prevalence of current smokers in New Zealand adults is $19.9 \%(18.8 \%$ of adult females are current smokers) [25]. Of the female population, $18.6 \%$ were regarded as 'ex-smokers' in the $2006 / 2007$ smoking survey $[26,27]$.

Of the 54 total participants, 35 (64.8\%) consumed alcohol at least once per week. Those in the IP group were more likely than those in the NP group to report the consumption of alcohol at least once per week. This was not significant $(P=0.10)$. According to Statistics New Zealand population indicators [28], there were $2,271,800$ females in the New Zealand population as of December 31, 2013. Of these, $43.2 \%$ consume alcohol at least once per week, as measured by the 2007/2008 New Zealand Alcohol and Drug Use Survey [29]. With these 
data, a Chi Square test for population proportions found that the 54 participants in this study were significantly more likely to consume alcohol at least once per week than the average New Zealand female $(P<0.001)$ which may be because there were few older women in the surgical sample.

\section{Employment and Education}

There was no statistically significant difference between the two baseline groups in terms of the proportions of participants employed (84.6\% NP vs. $78.6 \%$ IP, total $81.5 \% P=0.57)$. Similarly, there was no significant difference between the 'prolonged pain' group and the rest of the cohort $(P=0.401)$. The labor force participation rate reported by Statistics New Zealand for the June 2014 quarter was $68.5 \%$ [30].

A total of $34(63.0 \%)$ participants had received post-secondary education. This was not significantly higher for those 'in pain' $(P=0.180)$. Those who would go on to develop 'prolonged pain' were less (but not significantly) likely to have received postsecondary education $(45.5 \%$ vs. $64.4 \%$ $P=0.177$ ). In $2007,41 \%$ of all adults (aged 25-64 years) had received tertiary education (this included all post-secondary education) [31].

\section{DISCUSSION}

Of the 54 participants, $15.7 \%$ were deemed to be experiencing significant pain at 6 weeks postoperatively; $8.2 \%$ of participants were deemed to be experiencing significant pain at 3 months postoperatively. Of the psychometric questionnaires only the BIPQ appeared to be predictive of prolonged postoperative pain, while none of the other measures reached levels of significance.

\section{Prevalence of Pain}

Their SF-McGill scores on the sensory scale were significantly higher $(P<0.001)$ at 6 weeks compared to their baseline scores. This suggests that the pain experienced by these participants was unlikely to have simply been a symptom of their underlying condition, as it was qualitatively different. As such, it seems reasonable to assume this pain was largely the result of the surgery. Acute postoperative pain remains one of the most dependable and robust predictors of CPSP [32], especially the severity of acute postoperative pain in the days and weeks after surgery $[19,33]$. However, the investigator who conducted all interviews at all three points was not a gynecologist and, therefore, not qualified to determine if pain postoperatively was the result of surgery or not.

Of the 4 participants who were identified as experiencing clinically significant pain at 3 months following their surgery, only one of these was identified as in 'subacute' pain at the 6-week follow-up point. Two of the remaining 3 participants reported sub-threshold pain at that time of recording. Two of these 4 participants had been 'in pain' at the baseline assessment. Patients with preoperative pain related (e.g., in the anatomical region of the surgery) or not related (e.g., chronic low back pain) to the surgical site are at significantly greater risk of developing CPSP [18]. The presence of preoperative pain, as well as its intensity and duration remain risk factors for the development of early severe acute postoperative pain; this results in acute persistent pain in the days and weeks after surgery, and in CPSP $[19,33,34]$.

On the sensory scale of the SF-McGill, the scores of these 2 participants had changed sufficiently between baseline (4.6) and 
3 months (10.17) to consider them to be a likely effect of the surgery. As with those 'in pain' at 6 weeks, their affective scores did not differ from baseline to 3 months $(0.3-2.2)$. This is not the expected result, as it would seem logical that one would have to pass through the 'subacute' stage to reach a chronic pain state. However, as mentioned earlier, 2 of the remaining 3 participants had reported sub-threshold pain at the time of recording. Sub-threshold pain is either more important than previously thought, or the way sub-threshold pain was differentiated from 'above threshold' pain was not entirely accurate.

Given the small number 'in pain' at the follow-up points, the decision was made to analyze them $(n=11)$ as one 'prolonged pain' group. Seven participants $(63.6 \%)$ in this group had been 'in pain' at baseline. One $(9.1 \%)$ was 'in pain' at both 6 weeks and 3 months. It is worth noting that smaller $(n<300)$, singlecenter studies have found lower CPSP rates [35]; this may partially account for the low apparent prevalence.

Participants who were 'in pain' at baseline were slightly, but not significantly, more likely than those who were 'not in pain' to experience pain at either follow-up point (7 of 11 , or $63.6 \%, P=0.48)$. This suggests that those experiencing preoperative pain are more likely to develop postoperative pain that persists into a 'subacute' phase and then into a chronic phase. At an individual level though, this would not seem to be a strong predictive factor. In the absence of further evidence, the results of this study cannot support the clinical use of preoperative pain as a predictive factor.

\section{Psychometric Questionnaires}

High preoperative anxiety, surgical fear, and depression have been shown to predict severe postoperative pain $[19,36,37]$. In the 'in pain' group, baseline depression, anxiety, stress, and total DASS-21 scores were all higher than in the 'not in pain' group. This reinforces the association between pain and negative affect, even if no direction of causality can be directly established from this data. The effect of this higher level of psychological distress on perioperative clinical outcomes warrants further investigation. There was an overall drop in scores not only for the cohort as a whole, but also for those who had been deemed 'not in pain' at any of the three points. This indicates that pain relief was not the only factor involved in the improvement of affective scores.

Depression, anxiety, and stress sub-scale scores were associated with the pain state at all three follow-up points. DASS-21 scores in all sub-sections were higher in those who progressed into 'prolonged pain' states. This was not significant. It is possible the effect size that these scores can detect remains too small for the sample size (and too small to use clinically on individual patients). Another questionnaire on affect might have provided greater predictive value. Even in the absence of pain, it seems logical that thorough detection and management of these symptoms might improve the quality of life.

Other factors predicting severe postoperative pain that are identified less frequently include neuroticism, psychological vulnerability, avoidant coping, somatization, hypochondriasis, and a low expectation of return to work $[13,19]$. From the BIPQ results it looks as if those who go on to develop 'prolonged pain' after surgery have a more threatening view of their illness before their surgery than those who do not. This appears to conflict with the results of the PASS-20. Two of the PASS-20 subscales, escape and fearfulness, 
were even scored significantly lower at baseline by those who developed 'prolonged pain' than those who did not. This indicates that patients who go on to develop 'prolonged pain' states postoperatively identify their illness as particularly threatening. Pain catastrophizing has been shown to be a risk factor for CPSP after surgery $[19,37]$. However, they were no more emotionally distressed by their illness (or pain) than those who do not develop 'prolonged pain' states. This seems to be backed up (although not to levels of significance) by the sensory and affective scales of the SF-McGill. Those who went on to develop 'prolonged pain' states scored lower in these scales. In the PDI those who would develop 'prolonged pain' scored lower as well, but nearer to levels of significance. This suggests that these people are less disabled by their pain preoperatively. VAS pain scores at baseline did not differ significantly, nor were the raw means widely separated (the greatest being a mean difference of 0.8 for 'highest pain'). This makes it unlikely to become a clinically useful tool for predicting 'prolonged pain' states.

The only factor that could realistically be interpreted from these data as a potential 'screening'-type tool for the risk of developing 'prolonged pain' was the BIPQ. With a mean difference of 7.4 (95\% CI 0.01, 14.8, $P=0.049$ ) on a 0-50 scale, if a true difference, it would imply a mean of $14.9 \%$ higher scores in those at risk of developing 'prolonged pain'. While this is not a large enough difference to accurately predict each individual with the potential to develop 'prolonged pain', it provides a 'piece of the puzzle' to guide future clinical practice. More studies should investigate the role of the BIPQ in preoperatively predicting prolonged postoperative pain. Larger studies are needed to research the predictive abilities of each of the other questionnaires.
Any correlation of the characteristics measured by these questionnaires with lasting pain states implies that these symptoms should be detected and managed early. Simple tools such as the questionnaires used in this study could be used to screen preoperative patients. This would enable early detection and secondary prevention that would positively influence pain, psychological, and functional outcomes. In patients identified as having 'at risk' factors preoperatively, follow-up several weeks postoperatively could prove essential in the secondary prevention of chronic pain. This means intervening at the 'subacute' (or acute persistent pain) phase. Large controlled trials should investigate this.

\section{Demographic Information}

None of the demographic factors (age, ethnicity, education, work status, smoking status, and alcohol intake) or relevant histories (self-reported chronic pain, mental illness, or surgery) differed significantly between those who progressed into 'prolonged pain' states, and those who did not. The study was not sufficiently powered to detect the effects of these factors.

In terms of ethnicity, there were no significant differences between the groups at baseline. The cohort appeared to be in line with the 2013 New Zealand Census data for Canterbury. None of the participants experiencing 'prolonged pain' self-identified as New Zealand Māori. This is likely due to sample size, or under-reporting of pain by the New Zealand Māori participants. Younger age is one of the most consistently reported factors associated with an increased risk of development of CPSP $[19,34]$. Age was not a significant factor in predicting who would develop 'prolonged pain'. These results neither 
support nor reject the hypothesis that age influences the development of chronic pain. It is possible that the relationship between age and likelihood of developing 'prolonged pain' may not be linear. This study was undertaken using a group of patients largely over 30 years of age. As such, it cannot be concluded whether or not teenagers or younger adults would be at increased risk of developing 'prolonged pain' compared to other age groups and developmental periods. Participants who experienced 'prolonged pain' had increased rates of self-reported chronic pain, mental illness, weekly alcohol consumption, and former or current smoking. Their rates of employment and completed secondary education were lower as well. None of these results were significant despite following expected trends. Lower socioeconomic status and lower education and have been recognized as risk factors for CPSP as well [19, 34, 38]. The evidence remains mixed for other factors such as employment status, marital status, and for workers' compensation [19, 34, 38].

The participants in this study were all undergoing surgery. Repeated surgery in the same area is a known risk factor for the development of CPSP [19, 39]. A greater proportion of this cohort than would be expected from the limited evidence $(68.5 \%$ vs. $19-43.8 \%)[18,19]$ had undergone surgery in the past. Of particular note is that these sources stated lifetime risk, while the mean age of women in this study was only 41.5 years. This would point to previous surgery being a risk factor for requiring further surgery, whether through surgical failure or complications, recurrence of disease, or through CPSP. This study did not observe all relevant factors pertaining to the risk of requiring gynecological surgery. It is possible that there were other factors causing the observed association.

\section{Limitations}

Time limitations were caused by a delay of several months when for logistical reasons the project had to be redesigned. This resulted in fewer than the optimal number of participants being recruited. This combined with a patient group with lower rates of prolonged postoperative pain influenced the power of the study. Chronic pain is usually arbitrarily defined as pain lasting $>3$ or $>6$ months [40]. Time constraints forced the time point at which pain was defined as 'chronic' to be 3 months. This, however, had little effect. On the other hand, if chronic pain is defined as 'pain persisting beyond the accepted period of healing', 3 months is well beyond this point.

As highlighted earlier, the structure of the pre-admission clinic necessitated assistance from the nursing staff for the purposes of recruitment. Of course, their clinical duty to the patient came before the study. Some potentially eligible participants were 'shielded' by the nursing staff. From conversations with the staff, it seems these patients were largely the very anxious, and those with significant medical comorbidities. Given the correlation between psychological state and the experience of pain [41], it is likely that any sampling bias related to psychological distress would have impacted the pain scores at baseline, and potentially at the later follow-up points as well.

Some of questionnaires used were designed and only validated for use in chronic pain patients, so their accuracy for those in acute pain model has not been validated. In this study the associations between the depression/ anxiety scores and pain shows potential for the questionnaires to have a wider use than their current one. It was felt that specific questionnaires developed for pain would be 
more useful in a pain-based study than the more general screening questionnaires.

The observer was not clinically experienced in the field of gynecology. As a result, participants' reports of pain may have been inaccurately attributed to their surgery, when it is possible that for some participants' pain was unrelated to the surgery (such as a recurrence of their original condition). This was mitigated somewhat by comparing the subjective nature of the pain at each time point, using the information gathered by the SF-McGill. If the subjective nature of the pain was significantly different, it seems likely the cause of the pain was different as well. Loss to follow-up was not a large problem, with only five of fifty-four participants (9.3\%) dropping out. All five dropouts were unreachable by telephone despite multiple attempts to contact them. It is of note that four of these participants (and all the three lost at 6 weeks) had been identified as being 'in pain' at the baseline assessment; the remaining one reported an unspecified mental illness. This raises suspicion that those lost to follow-up at this point were potentially more likely to develop 'prolonged pain' states. Only eight participants (14.8\%) were identified as experiencing clinically significant pain at the 6-week follow-up point. Given the characteristics of those who were lost, it seems that this is an underestimate. The true number would probably lie between $14.8-20.4 \%$ (not an insignificant change).

\section{CONCLUSION}

At 6 weeks and 3 months postoperatively, 15.7\% and $8.2 \%$ of participants, respectively, experienced significant pain. The psychometric questionnaires used frequently detected differences between those 'in pain' and those 'not in pain' at given observation points. Only the BIPQ appeared predictive of developing prolonged postoperative pain. The mean difference (7.4 on a $0-50$ scale) should be enough to see it used clinically alongside other predictive measures. While none of the demographic factors observed significantly predicted the development of 'prolonged pain', the not significant data followed expected trends. Several relationships were detected in this study that should further efforts in developing preoperative predictors to promote the secondary prevention of postoperative pain states.

\section{ACKNOWLEDGMENTS}

No funding contribution was received towards selection, extraction or analysis of the data from any source. All named authors meet the International Committee of Medical Journal Editors (ICMJE) criteria for authorship for this manuscript, take responsibility for the integrity of the work as a whole, and have given final approval for the version to be published.

Conflict of interest. Campbell MacLachlan, Edward A. Shipton, and J. Elisabeth Wells declare no conflict of interests regarding the publication of this paper.

Compliance with ethics guidelines. Ethical approval was obtained from the University of Otago Human Ethics Committee (Health), and from the University of Otago, Christchurch Māori Research Advisor. Patients were approached at the Christchurch Women's Hospital preadmission clinic, where the study was explained to them, and written informed consent obtained if interest shown in participating in the study. 
Open Access. This article is distributed under the terms of the Creative Commons Attribution Noncommercial License which permits any noncommercial use, distribution, and reproduction in any medium, provided the original author(s) and the source are credited.

\section{REFERENCES}

1. Woolf CJ. Generation of acute pain: central mechanisms. Br Med Bull. 1991;47(3):534-8.

2. Merksey H, Bogduk N. Classification of chronic pain. Seattle: IASP Press; 1994.

3. Chanda M, Alvin MD, Schnitzer TJ, Apkarian AV. Pain characteristic differences between subacute and chronic back pain. J Pain. 2011;12(7):792-800.

4. Apkarian AV, Baliki MN, Geha PY. Towards a theory of chronic pain. Prog Neurobiol. 2009;87:81-97.

5. Kehlet H, Jensen TS, Woolf CJ. Persistent postsurgical pain: risk factors and prevention. Lancet. 2006;367(9522):1618-25.

6. Shipton EA. Recognition of the vocational practice of the scope of Pain Medicine in New Zealand. N Z Med J. 2013;126:1368.

7. Pogatzki-Zahn ASE. Predictors of chronic pain following surgery \& \#xB7; What do we know? Der Schmertz. 2010;24:517-33.

8. Neil MJ, Macrae WA. Post surgical pain-the transition from acute to chronic pain. Rev Pain. 2009;3(2):6-9.

9. Smith BH, Torrance N, Bennett MI, Lee AJ. Health and quality of life associated with chronic pain of predominantly neuropathic origin in the community. Clin J Pain. 2007;23(2):143-9.

10. Blyth FM, March LM, Cousins MJ. Chronic pain related disability and use of analgesia and health services in a Sydney community. Med J Aust. 2003;179:83-7.

11. Dominick C, Blyth F, Nicholas M. Patterns of chronic pain in the New Zealand population. $\mathrm{N} \mathrm{Z}$ Med J. 2011;124(1337):63-76.

12. Blyth FM, March LM, Brnabic AJ, Jorm LR, Williamson M, Cousins MJ. Chronic pain in Australia: a prevalence study. Pain. 2001;89(2-3):127-34.
13. Katz J, Pagé MG. Identification of risk and protective factors in the transition from acute to chronic post surgical pain. In: Lynch M, Craig K, Peng P, editors. Clinical pain management: a practical guide. Oxford: Wiley-Blackwell; 2010. p. $32-41$.

14. Macrae WA. Chronic pain after surgery. $\mathrm{Br} \mathrm{J}$ Anaesth. 2001;87:88-98.

15. Gerbershagen HJ. Transition from acute to chronic postsurgical pain. Physiology, risk factors and prevention. Schmerz (Berlin, Germany). 2013;27(1):81-93.

16. Rashiq S, Dick BD. Post-surgical pain syndromes: a review for the non-pain specialist. Can J Anaesth. 2014;61(2):123-30.

17. Nikolajsen L, Minella CE. Acute postoperative pain as a risk factor for chronic pain after surgery. Eur J Pain. 2009;3:29-32.

18. Shipton E. Transition from acute post surgical pain to chronic post surgical pain. Anaesth Intensive Care. 2011;39(5):824-36.

19. Shipton EA. The transition of acute postoperative pain to chronic pain: part 1-risk factors for the development of postoperative acute persistent pain. Trends Anaesth Crit Care. 2014;4:67-70.

20. Statistics New Zealand. Population estimates and projections. 2014. Available from: http://www.stats. govt.nz/browse_for_stats/population/estimates_ and_projections/pop-indicators.aspx.

21. Wells JE. Prevalence and severity across aggregated disorders Wellington: Ministry of Health; 2006.

22. Nunoo-Mensah JW, Rosen M, Chan LS, Wasserberg $\mathrm{N}$, Beart RW. Prevalence of intra-abdominal surgery: what is an individual's lifetime risk? Southern J Med. 2009;102(1):25-9.

23. Smith FJ, Holman CD, Moorin RE, Tsokos NS. Lifetime risk of undergoing surgery for pelvic organ prolapse. Obstet Gynaecol. 2010;116(5):1096-100.

24. Wu JM, Matthews CA, Conover MM, Pate V, Jonsson FM. Lifetime risk of stress urinary incontinence or pelvic organ prolapse surgery. Obstet Gynaecol. 2014;123(6):1201-6.

25. Cigarette smoking-The social report te puongo oranga tangata. Wellington: 2010.

26. Factsheet-Smoking statistics. Auckland. Available from: http://www.ash.org.nz/wp-content/uploads/ 2013/01/Factsheets/01_Smoking_statistics_ASH_NZ_ factsheet.pdf. 
27. Hewitt A, Holmes E. Tobacco Use in New Zealand: Key findings from the 2009 New Zealand tobacco use survey. Wellington: Ministry of Health; 2010.

28. Population indicators. Statistics New Zealand 2013. Available from: http://www.stats.govt.nz/browse for_stats/population/estimates_and_projections/ pop-indicators.aspx.

29. Alcohol use in New Zealand. Key results of the 2007/08 New Zealand alcohol and drug use survey. Wellington: Ministry of Health; 2009.

30. Key Labour Force Measures by Qualification, Age and Sex Statistics New Zealand 2014. Available from: http://nzdotstat.stats.govt.nz/wbos/Index. aspx?DataSetCode=TABLECODE7080.

31. Education Counts 2010. Available from: http:// www.educationcounts.govt.nz/indicators/main/ education-and-learning-outcomes/1903.

32. Powell R, Johnston M, Smith WC, King PM, Chambers WA, Krukowski Z, et al. Psychological risk factors for chronic post-surgical pain after inguinal hernia repair surgery: a prospective cohort study. Eur J Pain. 2012;16(4):600-10.

33. Clark H, Woodhouse LJ, Kennedy D. Strategies aimed at preventing chronic post-surgical pain: comprehensive perioperative pain management after total joint replacement surgery. Physiother Can. 2011;63:289-304.

34. VanDenKerkhof EG, Peters ML, Bruce J. Chronic pain after surgery: time for standardization? A framework to establish core risk factor and outcome domains for epidemiological studies. Clin J Pain. 2013;29(1):2-8.

35. Aasvang E, Kehlet H. Chronic postoperative pain: the case of inguinal herniorrhaphy. Br J Anaesth. 2005;95(1):69-76.

36. Lavand'homme P. The progression from acute to chronic pain. Curr Opin Anaesth. 2011;24(5):545-50.

37. Celestin J, Edwards RR, Jamison RN. Pretreatment psychosocial variables as predictors of outcomes following lumbar surgery and spinal cord stimulation: a systematic review and literature synthesis. Pain Med. 2009;10(4):639-53.

38. Brandsborg B, Nikolajsen L, Kehlet H, Jensen TS. Chronic pain after hysterectomy. Acta Anaesthesiol Scand. 2008;52(3):327-31.

39. Shipton E. Postoperative pain, persistent acute Pain. In: Schmidt R, Willis W, editors. Encyclopedic reference of pain. Berlin: Springer-Verlag; 2013. p. $3079-83$.

40. Andersson HI, Ejertsson G, Leden I, Rosenberg C. Chronic pain in a geographically defined general population: studies of differences in age, gender, social class, and pain localization. Clin J Pain. 1993;9(3):174-82.

41. Merskey H, Lau CL, Russell ES, Brooke RI, James M, Lappano S, et al. Screening for psychiatric morbidity. The pattern of psychological illness and premorbid characteristics in four chronic pain populations. J Pain. 1987;30:141-57. 